PROGRESS IN MEASUREMENTS OF THE GRAVITATIONAL BENDING OF RADIO WAVES USING THE VLBA

This article has been downloaded from IOPscience. Please scroll down to see the full text article. 2009 ApJ 6991395

(http://iopscience.iop.org/0004-637X/699/2/1395)

The Table of Contents and more related content is available

Download details:

IP Address: 128.206.162.204

The article was downloaded on 08/04/2010 at 19:55

Please note that terms and conditions apply. 


\title{
PROGRESS IN MEASUREMENTS OF THE GRAVITATIONAL BENDING OF RADIO WAVES USING THE VLBA
}

\author{
E. Fomalont ${ }^{1}$, S. Kopeikin ${ }^{2}$, G. LANYI ${ }^{3}$, AND J. Benson ${ }^{4}$ \\ ${ }^{1}$ National Radio Astronomy Observatory, Charlottesville, VA 22903, USA; efomalon@nrao.edu \\ ${ }^{2}$ Department of Physics \& Astronomy, University of Missouri, Columbia, MO 65211, USA; kopeikins@missouri.edu \\ 3 Jet Propulsion Laboratory, California Institute of Technology, Pasadena, CA 91109, USA; gabor.e.lanyi@jpl.nasa.gov \\ ${ }^{4}$ National Radio Astronomy Observatory, Socorro, NM 87801, USA; jbenson@nrao.edu \\ Received 2008 October 17; accepted 2009 May 5; published 2009 June 23
}

\begin{abstract}
We have used the Very Long Baseline Array (VLBA) at 43, 23, and $15 \mathrm{GHz}$ to measure the solar gravitational deflection of radio waves among four radio sources during an 18 day period in 2005 October. Using phase-referenced radio interferometry to fit the measured phase delay to the propagation equation of the parameterized post-Newtonian formalism, we have determined the deflection parameter $\gamma=0.9998 \pm 0.0003$ (68\% confidence level), in agreement with general relativity. The results come mainly from $43 \mathrm{GHz}$ observations where the refraction effects of the solar corona were negligible beyond 3 deg from the Sun. The purpose of this experiment is three-fold: to improve on the previous results in the gravitational bending experiments near the solar limb; to examine and evaluate the accuracy limits of terrestrial VLBI techniques; and to determine the prospects and outcomes of future experiments. Our conclusion is that a series of improved designed experiments with the VLBA could increase the presented accuracy by at least a factor of 4 .
\end{abstract}

Key words: gravitation - quasars: individual (3C279) - relativity - techniques: interferometric

Online-only material: color figure

\section{INTRODUCTION}

The parameterized post-Newtonian (PPN) formalism was designed by Nordtvedt and Will (Will 1993) to analyze plausible deviations in the general theory of relativity in the case of a spherically symmetric gravitational field of a massive body. The PPN formalism introduces several phenomenological parameters that are targets for experimental measurements, and the propagation of electromagnetic waves is affected strongly by one of them, $\gamma$. This parameter empirically measures the deviation from the linear approximation of general relativity in which case the value of $\gamma=1.0$ (Einstein 1916): The Newtonian limit in this linear approximation would imply a value $\gamma=0$ (see Equation (7.22) in Will 1993). The parameter $\gamma$ can be determined precisely and unambiguously by measuring the deflection of electromagnetic radiation by the gravitational field of the Sun, with the GR value of 1".750 at the solar limb. Such experiments have been performed since 1919 (Dyson et al. 1920), and the most accurate interferometric observations have shown that $\gamma$ is consistent with unity to an accuracy of less than one part in 1000 (Shapiro 1964; Fomalont \& Sramek 1976; Robertson et al. 1991; Lebach et al. 1995; Shapiro et al. 2004). Recently, Bertotti et al. (2003) have made use of the Cassini tracking data in 2002 to set the upper limit of $\gamma$ to a few parts in 100,000. Somewhat less accuracy has been obtained with another analysis of the data (Anderson et al. 2004), and there are some concerns about the effect of the motion of the solar barycenter (Kopeikin et al. 2007). Hence, additional accurate and independent measurements are important to determine the value of the fundamental parameter, $\gamma$, using the radio-interferometric and other techniques.

With the demonstrated accuracy of the Very Long Baseline Array (VLBA) to measure relative positions of radio sources to 0.01 mas (Fomalont \& Kopeikin 2003; Brunthaler et al. 2006), the gravitational bending could be measured potentially with the radio-interferometric technique to a few parts in 100,000, although the coronal refraction when observing within a few degrees of the Sun produces large path-length changes. In this paper, we present the results from the measurement of $\gamma$ performed with the VLBA in 2005 October, and we suggest how more accurate measurements of $\gamma$ can be obtained.

\section{THE OBSERVATIONS AND DATA REDUCTION}

\subsection{The Observational Strategy and Parameters}

Every year in early October, the Sun passes in front of the strong radio source $3 \mathrm{C} 279$. By measuring the change of its angular position, caused by the gravitational bending effect, with respect to other nearby sources over a period of several weeks, the gravitational parameter $\gamma$ can be accurately determined, and we used the opportunity in 2005 to conduct a dedicated VLBA experiment. In contrast to several previous interferometric experiments that measured the relative position of 3C279 with respect to 3C273 using the group delay (e.g., Lebach et al. 1995), our observations used the phase delay, a more accurate astrometric quantity, to measure the change of relative position of $3 \mathrm{C} 279$ with respect to that of several fainter sources within a few degrees in the sky.

For phase referencing observations among close sources, the choice of sources to observe must be balanced between two competing factors: the closer the radio sources are in the sky, the more accurately their relative positions can be determined (Fomalont 2005); the farther apart the radio sources are in the sky, the greater the relative gravitational deflection among them. The angular scale pertinent to this balance is related to the closest that successful source observations can be made near the Sun, about 2 deg. The sensitivity of the VLBA determines whether there are any sources within a few degrees of 3C279 that can be detected in order to measure their accurate positions. From an existing catalog of compact and relatively bright sources (Petrov et al. 2008), several candidates were found; after brief VLBA observations, three were chosen for this experiment, and their configuration in the sky is shown in Figure 1. The a priori positions and total flux densities of the sources are 
Table 1

A Priori Radio Source Position and Total Flux Density

\begin{tabular}{lcrrrr}
\hline \multicolumn{1}{c}{ Source } & \multicolumn{2}{c}{ R.A. } & Decl. & $S_{43 \mathrm{GHz}}$ & $\begin{array}{c}S_{23 \mathrm{GHz}} \\
(\mathrm{Jy})\end{array}$ \\
\hline 3C279 & $125611.166557 \pm 0.000013$ & $-054721.52467 \pm 0.00031$ & 11.22 & 12.75 \\
J1246-0730 & $124604.232100 \pm 0.000014$ & $-073046.57456 \pm 0.00031$ & 0.24 & 0.34 \\
J1248-0632 & $124822.975657 \pm 0.000016$ & $-063209.81737 \pm 0.00041$ & 0.16 & 0.19 & 0.50 \\
J1304-0346 & $130443.642255 \pm 0.000022$ & $-034602.55122 \pm 0.00065$ & 0.38 & 0.48 \\
\hline
\end{tabular}

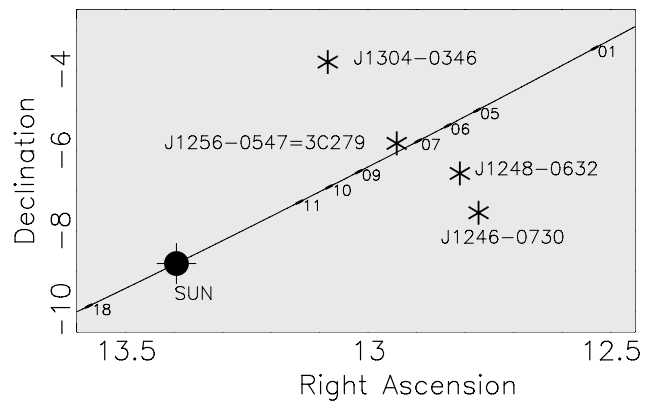

Figure 1. Source configuration for the deflection experiment: the solar trajectory between October 1 and 18 is shown by the diagonal line, with the eight observing days superimposed. The locations of the four radio sources are indicated.

shown in Table 1, and their positions, estimated uncertainties, and compactness have been determined within the International Celestial Reference Frame system to an accuracy of $<1$ mas. Even the weakest of the sources can be detected by the VLBA within about $20 \mathrm{~s}$ of integration time.

The sources were observed with the VLBA on 8 days (sessions) centered around the October 8 solar occultation of $3 \mathrm{C} 279$, October $1,5,6,7,9,10,11$, and 18, for a period of $6 \mathrm{hr}$ each session when the sources were above $10^{\circ}$ elevation at most of the VLBA antennas. Because the ionized plasma in the solar corona produces a frequency-dependent deflection that increases inversely with wavelength squared, observations at the highest routinely available VLBA frequency of $43 \mathrm{GHz}$ were chosen to minimize the coronal deflection. We also observed the sources at $23 \mathrm{GHz}$ and $15 \mathrm{GHz}$ in order to estimate the coronal deflection that was present.

Since the VLBA cannot observe simultaneously among 15 , 23 , or $43 \mathrm{GHz}$, it is not possible to remove the coronal refraction instantaneously. ${ }^{5}$ Thus, we had to choose a switching cycle among the sources and frequencies which minimized the effects of phase fluctuations from the troposphere and corona. Because the short-term tropospheric phase fluctuations at $43 \mathrm{GHz}$ can limit the coherence timescale to less than a minute (Beasley \& Conway 1995), it is crucial to switch observations (scans) among the sources within this period in order to keep the phase coherent between source scans. Since a change in the observing frequency at the VLBA takes at least $15 \mathrm{~s}$, any frequency switching would have considerably lengthened the time between scans, beyond the nominal coherence time. Thus, we decided to switch among the sources at one frequency with $40 \mathrm{~s}$ scans for about 20 minutes and then change frequencies and switch among the sources for another 20 minutes. This observing scheme is illustrated in

\footnotetext{
5 The 2.3/8.4 GHz simultaneous frequency system, which is available on the VLBA and used for most astrometric observations to remove the ionosphere refraction (Ma et al. 1998), is subjected to severe scattering by the coronal refraction within about $4 \mathrm{deg}$ of the Sun. Previous observations to measure the solar deflection at these low frequencies alone did not produce $\gamma$ accuracies better than the $10^{-3}$ level. Hence, higher frequencies were used (Lebach et al. 1995).
}

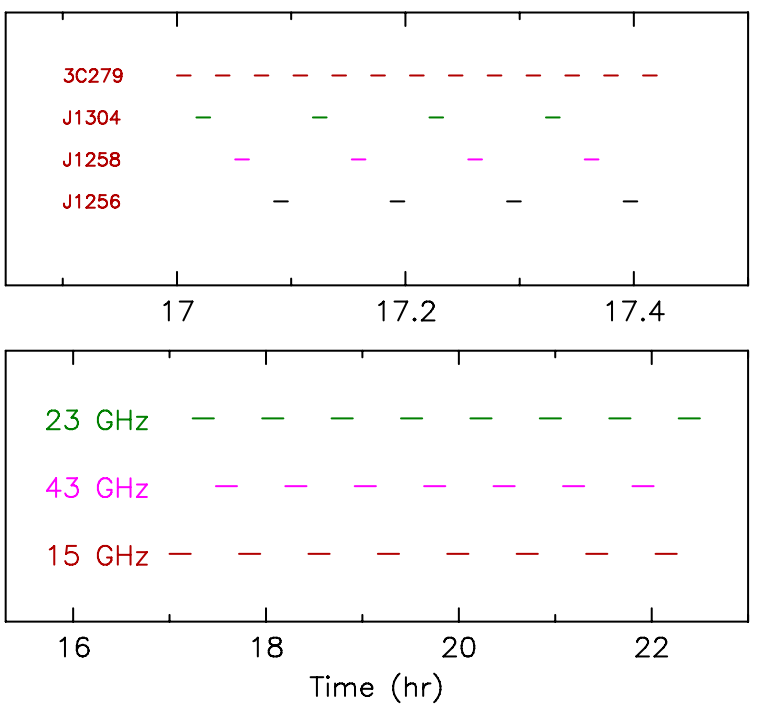

Figure 2. The observing strategy: the top plot shows the switching among the four sources within every 20 minute period. The bottom plot shows the switching of these 20 minute blocks among the three frequencies through each observing day.

(A color version of this figure is available in the online journal.)

Figure 2. Essentially, each 20 minute period of source switching can determine their accurate relative positions at one frequency. The slower cycling among the frequencies could determine the different positions among the frequencies caused by the coronal refraction, albeit on a relatively slow timescale of about $1 \mathrm{hr}$.

Typical VLBA observational setups are described by Walker (1995). At each of the three observing bands, we observed at four intermediate frequencies (IFs), each with a bandwidth of $16 \mathrm{MHz}$ : 14.93, 14.98, 15.20, 15.37 GHz; 23.02, 23.07, 23.29, $23.46 \mathrm{GHz} ; 42.73,42.78,43.00$, and $43.17 \mathrm{GHz}$. Each IF was separated into 16 frequency channels, each of width $1 \mathrm{MHz}$, and then sampled with two bits. The separation of the frequencies at each band permitted the determination of the group delay (phase slope versus frequency) to improve the tropospheric delay estimates using many calibrator observations at the beginning and end of each day. We also included several observations of the strong sources $3 \mathrm{C} 273$ and $\mathrm{J} 1310+3220$ over the day to monitor the instrumental delay changes. The observing schedule for the eight sessions was identical apart from slightly different start and stop times.

\subsection{Initial Data Calibration, Editing and Averaging}

The data from all antenna pairs were processed using the VLBA correlator in Socorro, NM, and the output visibility data were averaged into $2 \mathrm{~s}$ samples. The a priori model in the correlator uses the NASA CALC software package (http://www/gemini.gsfc.nasa.gov/solve) that includes the gravitational bending, assuming $\gamma=1$. Hence, the fitting parameter used in the data analysis is the departure of $\gamma$ from unity. 
(a) Tropospheric Phases on October 1

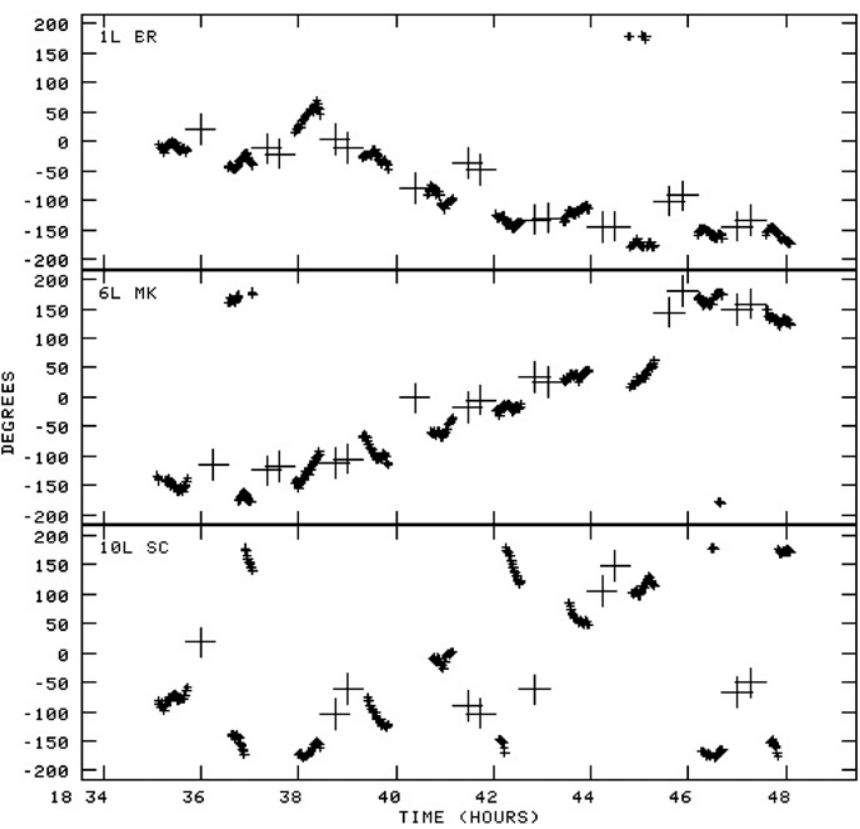

(b) Coronal Phases on October 9

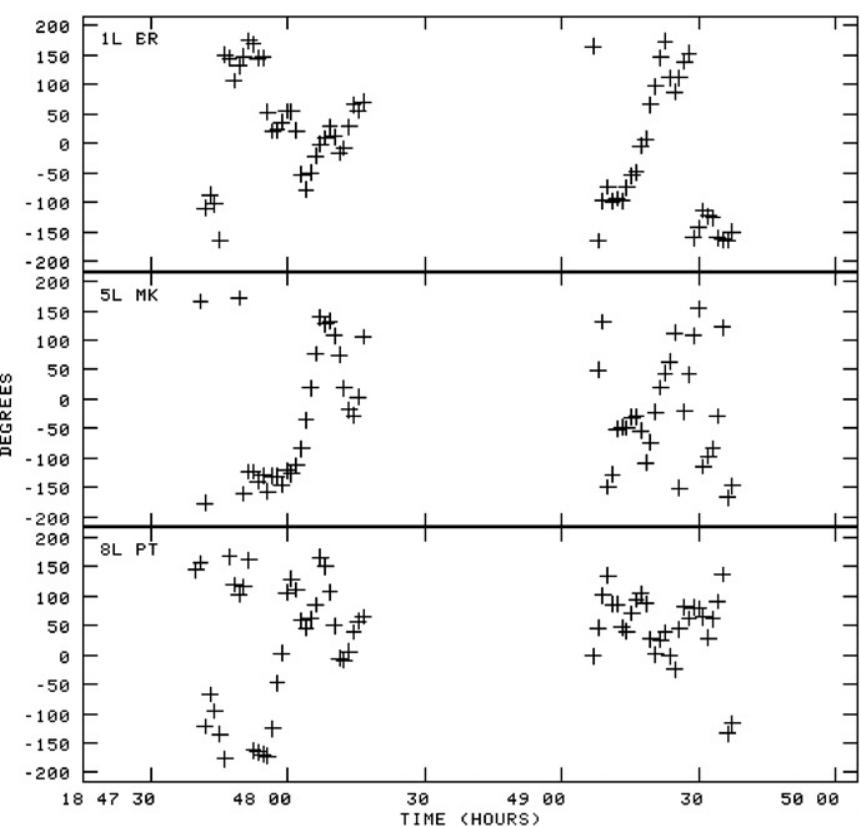

Figure 3. (a) Tropospheric phase : the phase behavior at $23 \mathrm{GHz}$ on October 1, when the sources were $>5^{\circ}$ from the Sun, is shown for three VLBA baselines from Los Alamos, NM (LA) to: Brewster, WA (BR), 1800 km; Mauna Kea, HI (MK), 5000 km; and Saint Croix (SC), VI, 4500 km. The period covers 13 minutes near the middle of the day, with the UT time given at the bottom. The densely packed points are the phases for $3 \mathrm{C} 279$ at $2 \mathrm{~s}$ intervals, and the more isolated larger points show the phases for the other calibrators, averaged over $20 \mathrm{~s}$ (two per scan). All sources lie on a relatively continuous temporal phase curve because their accurate relative positions were determined before producing the plot. (b) The coronal phase behavior: the phase behavior at $23 \mathrm{GHz}$ on October 9 for $3 \mathrm{C} 279$, when the source was 1.7 from the Sun, is shown for three VLBA baselines over a 2 minute period. The baseline between LA and Pie Town, NM (PT) is only $240 \mathrm{~km}$. Each plotted point shows the measured phase for $3 \mathrm{C} 279$ at $2 \mathrm{~s}$ intervals. The other signals from the other three sources observed during the gaps were too decorrelated to be detected.

The standard VLBA calibrations and editing were made on the correlated data (Ulvestad et al. 2001) independently for $15 \mathrm{GHz}$, $23 \mathrm{GHz}$, and $43 \mathrm{GHz}$ : (1) the a priori amplitude calibrations of each antenna, from the monitored system temperature and aperture efficiency, as well as the correlator bias, were applied; (2) data were flagged during receiver/antenna malfunctions, antenna mispointing, and when the source elevation for any antenna was below $10^{\circ}$; (3) improved parameters for the Earth orientation and rotation, polar motion, antenna motions, and nutation were available from online services and applied to the correlated data; (4) the calibrator observations at the beginning and end of each session were analyzed to determine corrections to the zenith path delay used in the correlator model (Mioduszewski \& Kogan 2005).

The data from the correlator for each observing frequency contained 64 streams: four IF frequencies, each split into 16 frequency channels. The relative gains and phases among the streams changed slowly and were determined using several observations in each session of strong sources, 3C273, and $\mathrm{J} 1310+332$ (and 3C279 when not too close to the Sun). The relative gain and phase determination among the frequency streams varied about $5 \%$ and $15^{\circ}$, respectively, over a session, so the changes were easily corrected. The 64 data streams were then coherently averaged to form one data stream with high signal-to-noise ratio. These data at $15 \mathrm{GHz}, 23 \mathrm{GHz}$, and $43 \mathrm{GHz}$ were then used to determine the shorter timescale phase changes induced by the troposphere and solar corona, described in the next section.

\subsection{Tropospheric Editing}

When observing at high radio frequencies, small "clouds" of water vapor pass through each antenna beam and produce variable delays of a few millimeters (about $100^{\circ}$ at $43 \mathrm{GHz}$ ) over timescales of a minute or less. These induced phase variations limit the astrometric and imaging accuracies. An example of relatively stable phase behavior is shown in Figure 3(a). Since $3 \mathrm{C} 279$ is a strong source, its phase can be determined for each $2 \mathrm{~s}$ sampled data point over a $40 \mathrm{~s}$ scan (closely spaced points), and over this period the typical phase variation over a scan is about $30^{\circ}$. In order to have coherent observations, the phase between the consecutive 3C279 scans must be unambiguously connected with no lobe ambiguities, otherwise the residual phase of the other sources (their measured phase minus the interpolated phase from 3C279) will be grossly in error. For the BR and MK baselines, the phase connection between scans of $3 \mathrm{C} 279$ is obvious, although residual phase offsets of $50^{\circ}$ do occur. However, for the SC baseline, the scan-to-scan phase changes of 3C279 are larger and in some cases (between 18:41 to 18:42) it is not clear whether the phase increased or decreased between scans, so this period with $\mathrm{SC}$ is of dubious astrometric quality.

Using plots similar to that of Figure 3(a) for all observations and frequencies, we edited periods when the phase coherence was poor. These periods often were associated with inclement weather conditions at an antenna site. The percentage of data removed was $4 \%$ at $15 \mathrm{GHz}, 7 \%$ at $23 \mathrm{GHz}$, and $17 \%$ at $43 \mathrm{GHz}$, mostly for the Saint Croix, VI and Hancock, NH antennas that are at relatively humid locations.

\subsection{Coronal Editing}

In Figure 3(b), we show a 2 minute segment of data at $23 \mathrm{GHz}$ for $3 \mathrm{C} 279$ on October 9 when the source was about 1.7 from the Sun. Although the phases are coherent over $2 \mathrm{~s}$, there are rapid changes of phase over $10 \mathrm{~s}$ or longer; hence the phase cannot be connected between scans. Notice that the phase changes for 
Table 2

Peak Flux Density Variations of Sources

\begin{tabular}{lrcrr}
\hline \hline Date/Frequency & \multicolumn{1}{c}{ 3C279 } & J1246 & J1248 & J1304 \\
\hline Oct $01: 43 \mathrm{GHz}$ & $10.1 \pm 0.3$ & $0.50 \pm 0.02$ & $0.40 \pm 0.02$ & $0.44 \pm 0.03$ \\
Oct $18: 43 \mathrm{GHz}$ & $9.0 \pm 0.4$ & $0.54 \pm 0.02$ & $0.48 \pm 0.02$ & $0.51 \pm 0.03$ \\
Oct $01: 23 \mathrm{GHz}$ & $12.9 \pm 0.2$ & $0.61 \pm 0.02$ & $0.41 \pm 0.03$ & $0.52 \pm 0.02$ \\
Oct $18: 23 \mathrm{GHz}$ & $11.2 \pm 0.4$ & $0.63 \pm 0.02$ & $0.50 \pm 0.04$ & $0.57 \pm 0.03$ \\
Oct $01: 15 \mathrm{GHz}$ & $13.7 \pm 0.7$ & $0.62 \pm 0.02$ & $0.28 \pm 0.02$ & $0.51 \pm 0.03$ \\
Oct $18: 15 \mathrm{GHz}$ & $12.9 \pm 0.3$ & $0.59 \pm 0.02$ & $0.27 \pm 0.02$ & $0.49 \pm 0.04$ \\
\hline
\end{tabular}

the shortest baseline (bottom plot) of $240 \mathrm{~km}$ are as variable as those for the longer baselines. This indicates that the coronal refraction significantly varies over second timescales with a linear scale-size smaller than a few hundred kilometers in the solar corona. ${ }^{6}$

We found that when a source was closer than $\sim 3^{\circ}$ from the Sun, phase-stable observations often were not possible because of the severe and short-term coronal refraction at all frequencies. Thus, all observations on October 6 and 7, when three of the four sources were within this angle from the Sun, were astrometrically worthless. In addition we discarded all data for $3 \mathrm{C} 279$ on October 5 and 9 and J1248 on October 5 because of excessive coronal turbulence.

\section{ASTROMETRIC ANALYSIS}

\subsection{The Removal of the Source Structure}

Although all of the sources are dominated by a compact core component, extended emission is also present and often associated with a jet component that emanates from the core. This structure causes two astrometric problems: the peak brightness of the core (best definition of the location of the source) is resolution dependent, and the structure may vary with time. We thus determined the source structures at each frequency on October 1 and October 18 using the self-calibration algorithm (Readhead \& Wilkinson 1978). Since this algorithm gives no positional information about the source, we arbitrarily placed the maximum brightness for each source at each frequency at the assumed a priori position of the source given in Table 1. This convention has no effect on the astrometric results since the analysis determines the residual true sky position of the peak of the source, relative to the assumed model and a priori position.

Because significant structure and intensity changes in most extragalactic source radio emission occur over months of time, little change was expected over the 17 day period of this experiment (Hughes et al. 1992). Changes in structure can also be indirectly inferred by the variability of the peak flux density of the sources that are given in Table 2. The only significant variability occurred for 3C279 and other monitoring ${ }^{7}$ showed that the source reached a maximum of $20 \mathrm{Jy}$ at $23 \mathrm{GHz}$ in early 2005 and decreased through the year. Hence, the decrease in flux density is real and the structure did change slightly over the experiment. Comparison of the accurate structures for 3C279 on October 1 and October 18 showed that minor variations occurred near the position 0.2 mas west and 0.1 mas east of the radio core at $43 \mathrm{GHz}$, but that the apparent position change of the peak brightness at $43 \mathrm{GHz}$ and $23 \mathrm{GHz}$ was less than 0.02 mas.

\footnotetext{
6 The analysis of the statistical properties of the phase data when the sources were close to the Sun can provide information on the velocity, density, and size of the coronal turbulent cells. This will be reported elsewhere.

7 http://www.vla.nrao.edu/astro/calib/polar/2005.
}

The source structures will be discussed and displayed in more detail elsewhere (Fomalont et al. 2009), but a summary follows: the sources J1246 and J1248 are nearly point sources at $43 \mathrm{GHz}$, with a faint component at $15 \mathrm{GHz}$ about 0.5 mas to the west and 1.4 mas to the northeast, respectively. The source $\mathrm{J} 1304$ is nearly double at $43 \mathrm{GHz}$ with a separation of 0.8 mas, and $3 \mathrm{C} 279$ has a jet component which extends about 1 mas southeast of the core.

Since the source structures were essentially unchanged over the 18 day period of the experiment, we removed their effects by dividing the visibility data with the visibility data model associated with the source structure (averaged from the October 1 and October 18 images) at each frequency. This produced a structure-free (effectively now a point source) data set for each source and frequency. Any resolution differences among the observing sessions will not produce a change in the location of the peak brightness of this revised data set.

\subsection{Phase Referencing to Obtain the Residual Phase}

After the initial calibrations, editing, and removal of the source structure, we then used the standard phase referencing technique on the visibility data to determine the relative position among the sources (Beasley \& Conway 1995). This technique uses one of the sources as the main reference to determine the phase error associated with each antenna for each reference source scan. This phase error is then interpolated between each reference scan, and applied to the other sources to obtain their residual phases. This technique is illustrated in Figure 3(a). Since the switching time and proximity in the sky among the sources were small, the phase errors associated with the reference source are virtually the same as that for all sources. Hence, any residual phases found for the non-calibrator sources are predominantly associated with a position offset from its a priori value. We used 3C279 as the main phase reference on October 1, 11, and 18 when it was not too close to the Sun, and J1304 as the main phase reference on other days. The phase referencing was done independently for each frequency.

\subsection{Source Position Determination from the Residual Phase}

Two analysis methods were used to determine the radio source positions from the measured residual phases for each source and frequency. The first method used the basic interferometric Fourier imaging and deconvolution techniques to produce an image of each source. We then determined the location of the peak brightness of the source (all nearly point sources since the structure component has been removed), and an error estimate based on the quality of the image and the signal-to-noise ratio at the peak intensity. For the second method, we fitted the measured residual phases directly to a point-source model and determined by a least-squares analysis the position offset from the a priori position and the error estimate based on the phase deviations from the best fit. 
Table 3

Source Positions with Respect to J1304

\begin{tabular}{|c|c|c|c|c|c|c|c|}
\hline \multirow{2}{*}{$\begin{array}{c}\text { Source } \\
\text { Date }\end{array}$} & \multirow{2}{*}{$\begin{array}{c}S_{R \odot} \\
(\mathrm{deg})\end{array}$} & \multicolumn{2}{|c|}{$43 \mathrm{GHz}$} & \multicolumn{2}{|c|}{$23 \mathrm{GHz}$} & \multicolumn{2}{|c|}{$15 \mathrm{GHz}$} \\
\hline & & $\mathrm{E} / \mathrm{W}$ & $\mathrm{N} / \mathrm{S}$ & $\mathrm{E} / \mathrm{W}$ & $\mathrm{N} / \mathrm{S}$ & $\mathrm{E} / \mathrm{W}$ & $\mathrm{N} / \mathrm{S}$ \\
\hline \multicolumn{8}{|l|}{$3 \mathrm{C279}$} \\
\hline Oct01 & 6.6 & $0.96 \pm 0.03$ & $-0.11 \pm 0.04$ & $0.77 \pm 0.03$ & $0.16 \pm 0.04$ & $0.78 \pm 0.04$ & $-0.05 \pm 0.05$ \\
\hline Oct05 & 2.8 & & & & & & \\
\hline Oct09 & 1.3 & & & & & & \\
\hline Oct10a & 2.2 & $0.95 \pm 0.10$ & $-0.09 \pm 0.14$ & $0.65 \pm 0.14$ & $-1.27 \pm 0.20$ & $0.36 \pm 0.23$ & $-2.30 \pm 0.30$ \\
\hline Oct10b & 2.4 & $0.93 \pm 0.13$ & $-0.17 \pm 0.18$ & $0.56 \pm 0.16$ & $-1.28 \pm 0.24$ & $0.20 \pm 0.28$ & $-2.80 \pm 0.40$ \\
\hline Oct11 & 3.2 & $1.03 \pm 0.03$ & $-0.11 \pm 0.04$ & $1.02 \pm 0.04$ & $-0.05 \pm 0.05$ & $1.11 \pm 0.07$ & $-0.29 \pm 0.09$ \\
\hline Oct18 & 10.2 & $0.93 \pm 0.02$ & $-0.08 \pm 0.03$ & $0.83 \pm 0.02$ & $0.16 \pm 0.04$ & $0.84 \pm 0.04$ & $-0.02 \pm 0.05$ \\
\hline \multicolumn{8}{|l|}{ J1246 } \\
\hline Oct01 & 5.5 & $0.75 \pm 0.05$ & $0.16 \pm 0.07$ & $0.64 \pm 0.05$ & $0.54 \pm 0.07$ & $0.81 \pm 0.05$ & $0.33 \pm 0.07$ \\
\hline Oct05 & 2.6 & $0.46 \pm 0.06$ & $0.15 \pm 0.10$ & $-0.23 \pm 0.08$ & $0.49 \pm 0.11$ & $-0.22 \pm 0.10$ & $2.15 \pm 0.14$ \\
\hline Oct09 & 3.7 & $0.76 \pm 0.05$ & $0.09 \pm 0.07$ & $0.82 \pm 0.07$ & $0.28 \pm 0.11$ & $0.78 \pm 0.05$ & $0.16 \pm 0.07$ \\
\hline Oct10 & 4.5 & $0.76 \pm 0.05$ & $0.09 \pm 0.07$ & $0.82 \pm 0.07$ & $0.28 \pm 0.11$ & $0.78 \pm 0.05$ & $0.16 \pm 0.07$ \\
\hline Oct18 & 12.1 & $0.71 \pm 0.05$ & $0.18 \pm 0.07$ & $0.68 \pm 0.05$ & $0.38 \pm 0.07$ & $0.86 \pm 0.05$ & $0.21 \pm 0.07$ \\
\hline \multicolumn{8}{|l|}{$\mathrm{J} 1248$} \\
\hline Oct01 & 5.3 & $0.70 \pm 0.05$ & $-0.40 \pm 0.07$ & $0.66 \pm 0.05$ & $-0.02 \pm 0.07$ & $0.85 \pm 0.05$ & $-0.15 \pm 0.07$ \\
\hline Oct05 & 1.8 & & & & & & \\
\hline Oct09 & 3.0 & $0.68 \pm 0.09$ & $-0.32 \pm 0.11$ & $0.80 \pm 0.18$ & $0.00 \pm 0.23$ & $1.02 \pm 0.10$ & $0.10 \pm 0.14$ \\
\hline Oct10 & 3.9 & $0.71 \pm 0.13$ & $-0.13 \pm 0.15$ & $0.65 \pm 0.13$ & $-0.19 \pm 0.19$ & $-0.59 \pm 0.21$ & $1.19 \pm 0.33$ \\
\hline Oct11 & 4.9 & $0.70 \pm 0.08$ & $-0.49 \pm 0.12$ & $0.74 \pm 0.10$ & $-0.40 \pm 0.15$ & $0.64 \pm 0.15$ & $-0.84 \pm 0.20$ \\
\hline Oct18 & 11.8 & $0.65 \pm 0.05$ & $-0.41 \pm 0.10$ & $0.67 \pm 0.05$ & $-0.25 \pm 0.07$ & $0.85 \pm 0.05$ & $-0.21 \pm 0.07$ \\
\hline
\end{tabular}

Although the relative source positions at each frequency could be derived from every 20 minute segment of data, the expected change of position during each session caused by the coronal bending and by any small departures of $\gamma$ from unity was estimated to be less than 0.20 mas at $15 \mathrm{GHz}$, and smaller at the higher frequencies. Hence, the data at each of the frequencies for an entire session could be analyzed with no significant position smearing. However, for $3 \mathrm{C} 279$ on October 10 when the coronal bending significantly changed, the analysis was split into two half-session periods.

Both methods used the same set of data, and they provided somewhat different positions and errors that are associated with the different effective weighting of the data points. For the source position, we used the average position obtained from the two methods. The methods provided three position errors, one from each analysis method and also one from the difference between the two position determinations. We chose the largest of the three as the error estimate. Finally, we adopted a floor uncertainty that was based on the theoretical signal-to-noise ratio of the data: 0.02 mas E/W and 0.03 mas N/S for 3C279, and $0.05 \mathrm{~ms} \mathrm{E} / \mathrm{W}$ and $0.07 \mathrm{mas} \mathrm{N} / \mathrm{S}$ for the other three sources.

The resulting source positions from the above analyses, made independently for each frequency, are shown in Table 3. In order to have a one fiducial reference point over the entire experiment, we have listed in Column 1 the relative positions of $3 \mathrm{C} 279$, $\mathrm{J} 1254$, and J1256 with respect to J1304 for all days. This requires only a trivial position translation to $\mathrm{J} 1304$ of the positions on days when $3 \mathrm{C} 279$ was used as the phase reference. ${ }^{8}$ The distance of the source from the Sun in degrees in mid-session is shown in column 2. When one or more of the sources were within 2.5 of the Sun, the coronal turbulence was often too severe to

\footnotetext{
8 Because the experimental errors are dominated by antenna-based residual tropospheric and ionospheric errors, rather than by receiver noise and other baseline-stochastic processes, there is no additional information by using the results from all six source pairs, compared with the non-degenerate set of the three source pairs listed.
}

obtain phase coherence and no positions could be determined. For this reason, there are no October 6 and 7 entries in the table. On October 10 when 3C279 was relatively close to the Sun, the analysis was made on the first half and second half of the session because of the relative large change in coronal refraction on this day.

For observations on October 1 and 18, when the sources were sufficiently far from the Sun, the maximum expected relative gravitational bending was 0.02 mas and coronal bending at $15 \mathrm{GHz}$ was 0.03 mas, less than the position errors for each day. Hence, the positions on these two dates were chosen to be the undeflected relative positions at each frequency among the sources. The position differences between the two days are generally consistent with the estimated errors, although there are several outliers are about $3 \sigma$, especially for the N/S positions for J1246 and J1248.

\subsection{Position Changes with Session and Frequency}

Table 4 lists the position changes from the non-deflected positions for the three sources with respect to $\mathrm{J} 1304$, as a function of session and frequency. The assumed non-deflected position is given on the first line for each source and frequency, and it is the weighted average of the October 01 and October 18 positions, given in Table 3 . The offsets of about 1 mas for $3 \mathrm{C} 279$, J1246, and $\mathrm{J} 1248$ reflect the error in the a priori position of J1304. The offset positions among $3 \mathrm{C} 279$, J1246, and J1248 are in good agreement and suggest that their a priori positions are consistent to about 0.3 mas. The slight differences in the offset positions among the three frequencies are real and show the effect of structure changes for each source among the frequencies. A more complete discussion of the registration of the source positions among the three frequencies is given elsewhere.

Below this line, the table then lists the position changes from the undeflected positions for the other observing dates. Since the gravitational bending with $\gamma=1$ between the sources 
Table 4

Deflection Changes Between the Sources and J1304

\begin{tabular}{|c|c|c|c|c|c|c|c|}
\hline \multirow[t]{2}{*}{ DATE } & \multirow[t]{2}{*}{ Type } & \multicolumn{2}{|c|}{$43 \mathrm{GHz}$} & \multicolumn{2}{|c|}{$23 \mathrm{GHz}$} & \multicolumn{2}{|c|}{$15 \mathrm{GHz}$} \\
\hline & & (mas) & $\mathrm{N} / \mathrm{S}$ & \multicolumn{2}{|c|}{ (mas) } & \multicolumn{2}{|c|}{ (mas) } \\
\hline \multicolumn{8}{|l|}{$3 C 279$} \\
\hline non-de & d position & 0.94 & -0.09 & 0.80 & 0.16 & 0.81 & -0.04 \\
\hline Oct10a & Each Freq & $0.00 \pm 0.10$ & $0.00 \pm 0.14$ & $-0.15 \pm 0.14$ & $-1.43 \pm 0.20$ & $-0.45 \pm 0.23$ & $-2.26 \pm 0.30$ \\
\hline Oct10a & Corona-Free & $0.06 \pm 0.11$ & $0.39 \pm 0.20$ & & & & \\
\hline Oct $10 b$ & Each Freq & $-0.01 \pm 0.13$ & $-0.08 \pm 0.18$ & $-0.24 \pm 0.16$ & $-1.44 \pm 0.24$ & $-0.61 \pm 0.28$ & $-2.76 \pm 0.40$ \\
\hline Oct $10 b$ & Corona-Free & $0.06 \pm 0.14$ & $0.33 \pm 0.25$ & & & & \\
\hline Oct11 & Each Freq & $0.08 \pm 0.03$ & $-0.02 \pm 0.04$ & $0.22 \pm 0.04$ & $-0.21 \pm 0.05$ & $0.30 \pm 0.07$ & $-0.25 \pm 0.09$ \\
\hline Oct11 & Corona-Free & $0.04 \pm 0.05$ & $0.03 \pm 0.04$ & & & & \\
\hline \multicolumn{8}{|l|}{$\mathrm{J1246}$} \\
\hline non-de & d position & 0.73 & 0.17 & 0.66 & 0.46 & 0.84 & 0.27 \\
\hline Oct05 & Each Freq & $-0.27 \pm 0.06$ & $-0.02 \pm 0.10$ & $-0.89 \pm 0.08$ & $0.03 \pm 0.11$ & $-1.06 \pm 0.10$ & $1.88 \pm 0.14$ \\
\hline Oct05 & Corona-Free & $-0.10 \pm 0.15$ & $-0.11 \pm 0.15$ & & & & \\
\hline Oct09 & Each Freq & $0.03 \pm 0.05$ & $-0.08 \pm 0.07$ & $0.16 \pm 0.07$ & $-0.18 \pm 0.11$ & $-0.06 \pm 0.05$ & $-0.11 \pm 0.07$ \\
\hline Oct09 & Corona-Free & $0.02 \pm 0.06$ & $-0.05 \pm 0.07$ & & & & \\
\hline Oct10 & Each Freq & $0.00 \pm 0.10$ & $0.19 \pm 0.13$ & $0.06 \pm 0.13$ & $-0.41 \pm 0.18$ & $0.11 \pm 0.18$ & $-0.34 \pm 0.31$ \\
\hline Oct10 & Corona-Free & $-0.02 \pm 0.11$ & $0.28 \pm 0.14$ & & & & \\
\hline Oct11 & Each Freq & $0.02 \pm 0.08$ & $-0.26 \pm 0.12$ & $0.00 \pm 0.10$ & $-0.17 \pm 0.14$ & $-0.09 \pm 0.15$ & $-0.09 \pm 0.10$ \\
\hline Oct11 & Corona-Free & $0.03 \pm 0.09$ & $-0.23 \pm 0.12$ & & & & \\
\hline \multicolumn{8}{|l|}{$\mathrm{J1248}$} \\
\hline \multicolumn{2}{|c|}{ non-deflected position } & 0.67 & -0.41 & 0.67 & -0.14 & 0.85 & -0.18 \\
\hline Oct09 & Each Freq & $0.01 \pm 0.09$ & $0.09 \pm 0.11$ & $0.13 \pm 0.18$ & $0.14 \pm 0.23$ & $0.17 \pm 0.10$ & $0.28 \pm 0.14$ \\
\hline Oct09 & Corona-Free & $-0.02 \pm 0.11$ & $0.05 \pm 0.11$ & & & & \\
\hline Oct 10 & Each Freq & $0.04 \pm 0.13$ & $0.28 \pm 0.15$ & $-0.02 \pm 0.13$ & $-0.05 \pm 0.19$ & $-1.44 \pm 0.21$ & $1.37 \pm 0.33$ \\
\hline Oct10 & Corona-Free & $0.09 \pm 0.16$ & $0.20 \pm 0.18$ & & & & \\
\hline Oct11 & Each Freq & $0.03 \pm 0.08$ & $-0.09 \pm 0.12$ & $0.07 \pm 0.10$ & $-0.26 \pm 0.15$ & $-0.21 \pm 0.15$ & $-0.66 \pm 0.20$ \\
\hline Oct11 & Corona-Free & $0.03 \pm 0.09$ & $-0.01 \pm 0.13$ & & & & \\
\hline
\end{tabular}

was included in the correlator model, the position changes will include only the gravitational bending associated with a $(\gamma-1)$ term, as well as that caused by noise and remaining tropospheric and coronal refraction components that differ among the sources.

The first line for an observing date, labeled "Each Freq", lists the residual positions of the sources for each of the three frequencies. All entries are obtained from the relevant entries in Table 3. The second line for an observing date, labeled "Corona Free", gives the residual coronal-free position. Since the coronal plasma deflection varies with the observation wavelength squared at radio frequencies (Thompson et al. 2001), the estimate of the coronal-free position, $\mathbf{p}_{\mathbf{0}}$, can be obtained from the relationship,

$$
\mathbf{p}\left(v_{i}\right)=\mathbf{p}_{0}+\mathbf{I} v_{i}^{-2}
$$

where $\mathbf{p}\left(v_{i}\right)$ are the measured positions $(x, y)$ at the three frequencies $v_{i}$, and $\mathbf{I}$ is the average coronal refraction (magnitude and direction in the sky) at $1 \mathrm{GHz}$ for the observation period (Rogers 1970). We determined the estimated values and uncertainty of the ionosphere correction and the corona-free position of the sources using a least-squares fit. The discussion of the effectiveness in obtaining corona-removed solutions is given in the next section.

\section{THE DETERMINATION OF THE PARAMETER $\gamma$}

The approximate accuracy for the determination of $\gamma$ from Table 4 can be estimated in a straightforward manner. Since the typical position uncertainty of one measurement (one source pair per day) is $\sim 0.07$ mas for the differential gravitational bending of $\sim 150$ mas, ${ }^{9}$ the measurement accuracy of 1 part in 2000 corresponds to an accuracy of $\gamma$ of 1 part in 1000. Since about 20 independent measurements of the bending were made (10 source pairs with two position coordinates), a sensitivity of $\gamma$ to a few parts in $10^{-4}$ is expected.

The more formal determination of the optimum value of $(\gamma-$ 1) from the experimental results in Table 4 is straightforward. The analysis minimizes the (normalized) chi-squared expression

$$
\chi_{k}^{2}=\frac{1}{k} \sum_{d, i}\left(\frac{P_{d}(i)-0.5(\gamma-1) D_{d}(i)}{\sigma_{d}(i)}\right)^{2},
$$

where $P_{d}(i)$ and $\sigma_{d}(i)$ are the measured position offset and error estimate, respectively, from Table 4 . The (i) loops over the sources (3C279, J1246, J1248), and the (d) loops over each day or half-day observation (Oct05, 09, 10a, 10b, 11). The term $D_{d}(i)$ is the differential general relativity gravitational bending prediction, averaged over the session. The sum is made over the $\mathrm{E} / \mathrm{W}$ and $\mathrm{N} / \mathrm{S}$ values and estimated errors separately, so the number of degrees of freedom is $k=19$ if all 10 observing points are used.

The results for several solutions for $\gamma$ using the different data sets from Table 4 are listed in Table 5. The normalized $\chi^{2}$ indicates the ratio of the rms of the best fit divided by that expected from the estimate error of each entry. The $43 \mathrm{GHz}$ corona-free fit has the lowest $\chi^{2}$ for two reasons: the lessening of some coronal effects, and the increase of the position errors. The two $43 \mathrm{GHz}$ only solutions (with no removal of the ionosphere

\footnotetext{
9 For example, on October 11 the average relative deflection between 3C279 and J1304 was 100 mas and 66 mas in the $\mathrm{E} / \mathrm{W}$ and N/S directions, respectively.
} 
Table 5

Solutions for $\gamma$

\begin{tabular}{lccc}
\hline \hline \multicolumn{1}{c}{ Solution Type } & $\gamma-1$ & $\sigma_{\gamma}$ & $\chi_{k}^{2}$ \\
& $10^{-4}$ & $10^{-4}$ & \\
\hline $43 \mathrm{GHz}$ data (corona-free) & -2.4 & 3.2 & 0.9 \\
$43 \mathrm{GHz}$ data only & -1.0 & 2.6 & 2.2 \\
$43 \mathrm{GHz}$ data only-Oct05 & -3.2 & 2.8 & 1.1 \\
$23 \mathrm{GHz}$ data only-Oct05 & -2.0 & 2.4 & 4.7 \\
\hline
\end{tabular}

contribution) show the effect of the Oct05 session that was made relatively close to the Sun. Finally, the $23 \mathrm{GHz}$ only solution has a relatively large normalized $\chi^{2}$, even excluding the Oct05 session, and suggests that coronal refraction, which is four times larger than that at $43 \mathrm{GHz}$, is dominating the sensitivity of the experiment at $23 \mathrm{GHz}$. Nevertheless, the variation of $\gamma$ and its estimated error among the different solutions are in good agreement and suggest that the determination of $\gamma$ changes little between several different analyses of the data.

\section{RESULTS AND DISCUSSION}

The solutions in Table 5, using different selections of data, are all consistent with the GR value of the parameter $\gamma=1$. For the results in this paper, we have taken an average of the four solutions to obtain $\gamma=0.9998 \pm 0.0003$. This result is a factor of three times more accurate than the previous dedicated radio interferometric observations made specifically to measure the gravitational bending: $0.9996 \pm 0.0017$ (Lebach et al. 1995), and $1.0002 \pm 0.0010$ (Robertson et al. 1991). Using the radio geodetic database of thousands of observing sessions between 1979 and 1999, Shapiro et al. (2004) obtained $\gamma=$ $0.9998 \pm 0.0004$, comparable in accuracy to the present results. The result from the measurements as the spacecraft Cassini passed by the Sun in 2002 September is $\gamma=1.00002 \pm 0.00002$ (Bertotti et al. 2003), and was discussed in Section 1.

Although expected departures from $\gamma=1$ are likely to be a factor of 10 to 100 smaller than the accuracy of this experiment, some possibilities are: (1) the long-range scalartensor interaction in scalar-tensor theories of gravity (Will 1993) and Damour \& Nordtvedt (1993) predict a lower bound for the present value of $\gamma$ at the level of $10^{-6} \sim 10^{-7}$; (2) the long-range vector-tensor interaction in vector-tensor theories of gravity (Kostelecký 2004) may suggest a "spontaneous violation" of the Lorentz invariance and could modify the value of $\gamma$ (Bailey \& Kostelecký 2006); (3) the more complicated nature of the gravitational coupling between the curvature and stress-energy tensor of matter may lead to changes of $\gamma$ (Jaekel \& Reynaud 2005); (4) the plausible existence of the effective graviton's mass (Babak \& Grishchuk 2003) that would avoid the van Dam-Veltmann-Zakharov discontinuity (van Dam \& Veltman 1970; Zakharov 1970) would also effect the value of $\gamma$.

There are several changes in the design of a similar VLBA experiment that should improve the accuracy of $\gamma$ by about a factor of 2. First, by choosing a set of sources that can be observed when the Sun is further north, ${ }^{10}$ each day's integration time can be increased from 6 to $10 \mathrm{hr}$, and the sources will be on average at higher elevations than those from the present

\footnotetext{
10 Several possible experiments are: March 30 to April 24-J0121+1149, J0104+1134, J0139+0842, J0129+1146; April 10 to May 01-J0204+15114, J0158+1307, J0209+1352, J0211+1051.
}

experiment. We estimate that a more northern experiment will lower the position rms by about $20 \%$.

Second, we lost some sensitivity in the determination of $\gamma$ by not observing on days when the gravitational deflection was relatively large (e.g., October 3, 4, 12, and 13). We found that the most accurate results are obtained between solar distances of 3-5 deg, corresponding to about 3 days to 7 days from the nearest approach of the sources with the Sun. Thus, the addition of the above four observing days in the 2005 experiment would have decreased the position rms by $25 \%$.

Finally, the 2005 experiment devoted too much observation time at $15 \mathrm{GHz}$ and possibly $23 \mathrm{GHz}$ in an attempt to lessen the effect of coronal bending. When the coronal bending was significant at $43 \mathrm{GHz}$ ( $>0.1$ mas), the phase stability degraded considerably at all frequencies and no astrometric information could be obtained. Thus, future observations should concentrate on $43 \mathrm{GHz}$, somewhat further from the Sun. Additional observations at $23 \mathrm{GHz}$ are useful to monitor the coronal refraction since it can be variable with time, but observations at $15 \mathrm{GHz}$ are not helpful in determining the coronal refraction. The potential additional observing time by a factor of 2 at the primary frequency of $43 \mathrm{GHz}$ in a new experiment, compared with the 2005 experiment, would decrease the position rms by about $30 \%$.

Hence, the above three changes of strategy in a VLBAdesigned experiment will clearly improve the accuracy of the determination of $\gamma$ from a single 10 day experiment by at least about a factor of 2 compared with that of the 2005 October experiment. The availability of many such experiments over the year, all repeatable from year to year, can provide independent results estimates of $\gamma$ since the dominant error is produced by the quasi-random errors of the short-term troposphere and, to a lesser extent, coronal refraction. Also, any small systematic errors associated with the different sky configuration for the sources among the other possible experiments should be somewhat independent. Thus, we expect that the accuracy of $\gamma$ should improve by roughly the square root of the number of experiments. Hence, with sufficient VLBA resources, the uncertainty in the parameter $\gamma$ can be decreased by at least a factor of 4 compared with the results given in this paper.

The National Radio Astronomy Observatory is a facility of the National Science Foundation operated under cooperative agreement by Associated Universities, Inc. Research performed at the Jet Propulsion Laboratory is supported by a NASA contract with the California Institute of Technology.

\section{REFERENCES}

Anderson, J. D., Lau, E. L., \& Gaipieri, G. 2004, Texas Symp. on Relative Astrophysics at Standford Univ. 2004 December 13-17, http://www.slac.stanford.edu/econf/C041213/papers/0305.PDF

Babak, S. V., \& Grishchuk, L. P. 2003, Int. J. Mod. Phys. D, 12, 1905

Bailey, Q. G., \& Kostelecký, V. A. 2006, Phys. Rev. D, 74, 045001

Beasley, A. J., \& Conway, J. E. 1995, in ASP Conf. Ser. 82, Very Long Baseline Interferometry and the VLBA, ed. J. A. Zensus, P. J. Diamond, \& P. J. Napier (San Francisco, CA: ASP), 328

Bertotti, B., Iess, L., \& Tortora, P. 2003, Nature, 425, 374

Brunthaler, A., Reid, M. J., Falcke, H., Greenhill, L. J., \& Henkel, C., Science, 307,1440

Damour, T., \& Nordtvedt, K. 1993, Phys. Rev. D, 48, 3436

Dyson, F. W., Eddington, A. S., \& Davidson, C. 1920, Phil. Trans. R. Soc. A, 220,291

Einstein, A. 1916, Ann. Phys., 49, 769 
Fomalont, E. B. 2005, in ASP Conf. Ser. 340, Future Directions in High Resolution Astronomy: The 10th Anniversary of the VLBA, ed. J. Romney \& M. Reid (San Francisco, CA: ASP), 460

Fomalont, E. B., \& Kopeikin, S. M. 2003, ApJ, 598, 7044

Fomalont, E., Kopeikin, S., Lanyi, G., \& Benson, J. 2009, AJ, submitted

Fomalont, E. B., \& Sramek, R. A. 1975, Phys. Rev. Lett., 36, 1475

Hughes, P. A., Aller, H. D., \& Aller, M. F. 1992, ApJ, 396, 469

Jaekel, M.-T., \& Reynaud, S. 2005, Class. Quantum Gravity, 22, 2135

Kopeikin, S. M., Polnarev, A. G., Schäfer, G., \& Vlasov, I. Yu. 2007, Phys. Lett. A, 367, 276

Kostelecký, V. A. 2004, Phys. Rev. D, 69, 105009

Lebach, D. E., Corey, B. E., Shapiro., I. I., Ratner, M. I., Webber, J. C., Rogers, A. E. E., Davis, J. L., \& Herring, T. A. 1995, Phys. Rev. Lett., 75, 1439

Ma, C., et al. 1998, AJ, 116, 516

Mioduszewski, A. J., \& Kogan, L. 2004, in AIPS Memo 110, Strategy for Removing Tropospheric and Clock Errors using DELZN., http:// www.aoc.nrao.edu/aips/aipsmemo.html

Petrov, L., Kovalev, Yu. Y., Fomalont, E., \& Gordon, D. 2008, AJ, 136, 580
Readhead, A. C. S., \& Wilkinson, P. N. 1978, ApJ, 223, 25

Robertson, D. S., Carter, W. E., \& Dillinger, W. H. 1991, Nature, 349, 768

Rogers, A. E. E. 1970, Radio Sci., 5, 1291

Shapiro, I. I. 1964, Phys. Rev. Lett., 13, 789

Shapiro, S. S., Davis, J. L, Lebach, D. E., \& Gregory, J. S. 2004, Phys. Rev. Lett., 92, 121101

Thompson, A. R., Moran, J. M., \& Swenson, G. W. Jr. 2001, in Inteferometry and Synthesis in Radio Astronomy, 2nd edn (New York: Wiley), 557

Ulvestad, J., Greisen, E. W., \& Mioduszewski, A. J. 2001, in AIPS Memo 105, AIPS Procedures for Initial VLBA Data Reductions, http://www.aoc.nrao. edu/aips/aipsmemo.html

van Dam, H., \& Veltmann, M. 1970, Nucl. Phys., 22, 397

Walker, R. C. 1995, in ASP Conf. Ser. 82, Very Long Baseline Interferometry and the VLBA, ed. J. A. Zensus, P. J. Diamond, \& P. J. Napier (San Francisco, CA: ASP), 247

Will, C. M. 1993, Theory and Experiment in Gravitational Physics (Cambridge: Cambridge Univ. Press)

Zakharov, V. I. 1970, JETP Lett., 12, 312 